\title{
Impfpflicht—jetzt also doch?
}

Cornelia Betsch ${ }^{1,2}$, Philipp Sprengholz ${ }^{1}$, Philipp Schmid ${ }^{1}$, Lars Korn ${ }^{1,2}$, Sarah Eitze ${ }^{1}$, Pia Ochel $^{1}$, Lisa Felgendreff ${ }^{1}$, Elena Engel ${ }^{1} \&$ Robert Böhm $^{3,4}$

1 Universität Erfurt | 2 Bernhard-Nocht-Institut für Tropenmedizin, Hamburg | 3 Universität Wien 4 Universität Kopenhagen

Kontakt: cornelia.betsch@uni-erfurt.de

\section{Zusammenfassung}

Trotz aktuell steigender Impfbereitschaft unter den Erstimpfungen ist nicht abzusehen, dass die vom RKI geforderten Impfquoten für die künftige Kontrolle des SARS-CoV-2 Virus mit den bisherigen Maßnahmen zur Steigerung der Impfbereitschaft erreicht werden können (Stand: Ende November 2021). Daher werden in diesem Artikel drei Typen verpflichtender Regelungen vorgestellt (Terminpflicht, Beratungspflicht, Impfpflicht) und die Vorteile und Nachteile einer Impfpflicht aufgeführt.

Vorteile einer Impfpflicht

- $\quad$ Eine Impfpflicht ist sozial und stärkt das Gemeinwohl

- Eine Impfpflicht kann effektiv sein

- Eine Impfpflicht gegen COVID ist aktuell akzeptiert

- Eine Impfpflicht kann sich positiv auf die Gesellschaft auswirken

- Eine Impfpflicht kann Vertrauen stärken

Nachteile einer Impfpflicht

- Eine Impfpflicht entbindet nicht von der Aufklärung

- Eine Impfpflicht kann psychologische Nebenwirkungen haben

- Eine zielgruppenspezifische Impfpflicht könnte die Gesamt-Impfquote negativ beeinflussen

- Eine Impfpflicht könnte "impffaul" machen

- Eine Impfpflicht braucht begleitende Maßnahmen zur Stärkung der Akzeptanz

Als Wissenschaftler:innen aus Psychologie und Gesundheitskommunikation setzen wir uns für eine informierte, evidenzbasierte Impfentscheidung ein und befürworten eine Impfpflicht nicht grundsätzlich. Aufgrund der zu erwartenden hohen gesundheitlichen und gesellschaftlichen Kosten und Belastungen, die das Fehlen einer Impfpflicht und damit eine zu niedrige Impfquote mit sich bringt, empfehlen wir jedoch nun, umgehend Beratungen über das Design, die Umsetzung, die rechtliche Grundlage und die Abfederung möglicher Folgen zu beginnen. Hierfür sollte ein interdisziplinär besetztes Expert:innen-Team herangezogen werden, in dem auch Sozial- und Verhaltenswissenschaftler:innen sowie Bürger:innen vertreten sind.

Die Autor:innen erklären, dass keine Interessenskonflikte vorliegen. 


\section{5 nach 12 und zu wenige sind geimpft}

Bei 8 bis 9 von 10 Ansteckungen ist mindestens eine Person ohne Impfschutz involviertentweder als Ansteckende:r, als Angesteckte:r, oder meistens beides (Maier et al., in Vorbereitung). Die Infektionszahlen sind vor allem in Regionen Deutschlands sehr hoch, in denen die Impfquote niedrig ist. Die Impfbereitschaft der noch ungeimpften Personen ist just in diesen Teilen Deutschlands auch geringer als in den Regionen mit bereits hoher Impfquote (COSMO \#56). Zwar steigt aktuell die Impfbereitschaft auch unter Ungeimpften minimal an-jedoch ist über die Hälfte der aktuell Ungeimpften "auf keinen Fall” zu einer Impfung bereit (COSMO \#56). Selbst wenn sich alle bislang nicht geimpften Personen sofort impfen ließen, würde es aktuell zu lange dauern, um die vierte Welle effektiv zu brechen. Andere Maßnahmen werden hier notwendig sein. Dennoch wird auch nach der vierten Welle eine hohe Impfquote notwendig sein, um weitere Wellen zu verhindern. Um also nicht wie bei einem Jo-Jo-Effekt immer wieder zwischen harten und lockeren Maßnahmen zu wechseln, ist auch langfristig eine sehr hohe Impfquote notwendig-laut RKI 85\% der 12-59-Jährigen und 90\% der Über-60-Jährigen (Wichmann et al., 2021) —wenn nicht auf eine Durchseuchung mit allen kurz- und langfristigen Folgen gesetzt werden soll.

\section{Wortbruch und Meinungsänderung}

Politiker:innen vieler Parteien haben sich öffentlich bereits früh in der Pandemie gegen eine Impfpflicht ausgesprochen und mögen ein Umschwenken jetzt als Wortbruch wahrnehmen. Jedoch haben sich inzwischen viele Aspekte in der Pandemie geändert und eine Anpassung der eigenen Haltung zeigt Stärke und kann auch verlorengegangenes Vertrauen wieder erhöhen (WHO Regional Office for Europe, 2017). Die bereits geimpften Personen sind auch die, die die Maßnahmen zur Pandemiebekämpfung mittragen. Diese Gruppe hat aktuell höheres Vertrauen in die Politik als ungeimpfte Personen. Durch langsames Handeln bei rasant steigenden Fallzahlen sinkt aktuell aber auch deren Vertrauen (COSMO \#56). Die Einführung einer effektiven Infektionsschutzmaßnahme könnte also bei dieser großen Gruppe, der Mehrheit, das beschädigte Vertrauen wieder reparieren.

Viele der Forschungsarbeiten aus unserer Arbeitsgruppe haben ebenfalls bislang eine ablehnende Haltung zur Impfpflicht unterstützt - so haben wir kritische Stellungnahmen zur Masernimpfpflicht verfasst (z.B. hier) und uns auch in der Corona-Pandemie bislang kritisch zu einer möglichen Impfpflicht geäußert. Wir verfolgen mit großer Überzeugung das Ziel der guten Gesundheitsaufklärung und die Unterstützung einer informierten, autonomen und wissenschaftsbasierten Entscheidung. Als inhaltliches Hauptargument gegen die Impfpflicht haben wir außerdem die psychologischen Nebenwirkungen einer Impfpflicht aufgeführt: eine Impfpflicht kann bei Personen, die Impfen ablehnen, aufgrund von Reaktanz (Ärger) zu "Trotzverhalten" führen — und sich negativ auf andere Impfentscheidungen auswirken. Andere freiwillige Impfungen können dann möglicherweise ausgelassen werden als Zeichen des Widerstands und um die eingeschränkte Entscheidungsfreiheit wieder herzustellen (Betsch \& Böhm, 2016; Omer et al., 2018; Sprengholz et al., 2021a, 2021b).

Die aktuelle Lage — hohe Fallzahlen, zu niedrige Impfquote, hoher Anteil von Personen, die sich nicht impfen lassen wollen-führen jedoch auch bei uns zu einer Neubewertung, 
weswegen wir hier Gründe für und gegen eine Impfpflicht in den Kontext der aktuellen Situation stellen möchten, um sie so der öffentlichen Diskussion und Entscheider:innen zur Verfügung zu stellen. Alle bisherigen, meist digitalen Kampagnen, \#ärmelhoch der Institutionen, \#impfenschützt der Konzertmusiker:innen und zuletzt \#allesindenarm auf Twitter konnten trotz der Aufrufe berühmter Personen, aufklärenden Videos von Youtuber:innen und Informationsmaterial in vielen Sprachen nicht zu einer ausreichenden Erhöhung der Impfbereitschaft beitragen. Auch zeitweise niedrigschwellige Angebote durch Impfzentren, Impfbusse, mobile Impfteams und Impfaktionen an ungewöhnlichen Orten haben möglicherweise für ein schnelleres Erreichen eines Plateaus, nicht aber eine ausreichende Erhöhung der Impfquote gesorgt.

\section{Die aktuelle Impfbereitschaft gegen COVID-19}

Die Impfbereitschaft der aktuell ungeimpften Personen ist niedrig - immer noch mehr als die Hälfte möchte sich "auf keinen Fall” impfen lassen (COSMO \#56), auch wenn dieser Anteil wahrscheinlich angesichts aktuell rasant steigender COVID-Fallzahlen etwas geringer wird. Andere Studien und Umfragen zeigen ähnliche Ergebnisse (COVIMO, FORSA). Es ist unwahrscheinlich, dass Appelle an die Eigenverantwortung oder leichte bis mittlere Einschränkungen für Ungeimpfte im Sinne einer 3G- oder 2G-Regel zu einer deutlichen Steigerung führen können. Durch Veränderung in den Corona-Maßnahmen hat sich auch zuletzt kaum etwas am Anteil derer verändert, die sich auf keinen Fall impfen lassen wollen (COSMO \#56). Aktuell ist der wichtigste Grund für die Ablehnung der Impfung das fehlende Vertrauen in die Sicherheit der Impfung. Hierzu bedarf es - mit oder ohne Impfpflichtschnellstens bester Aufklärung, um Menschen die Angst vor der Impfung zu nehmen.

\section{Impfpflicht jetzt klug designen}

Verpflichtende Regelungen können unterschiedlich ausgestaltet werden. Verschiedene Schritte innerhalb der Entscheidung können verpflichtend sein (z.B. der Erhalt eines Termins, die Beratung oder die Impfung selbst), unterschiedliche Ausnahmen können erlaubt werden (z.B. aus medizinischen, religiösen oder weltanschaulichen Gründen) und Konsequenzen einer Verletzung der Pflicht können unterschiedlich ausfallen (z.B. Ausschluss von Veranstaltungen, Arbeitsverbot, Geldstrafe). Einzelne Varianten werden im Folgenden vorgestellt. Für alle gilt: Nicht-Impfen sollte mindestens ebenso aufwändig sein wie Impfen.

\section{Terminpflicht: Impftermine werden automatisch zugesandt}

Sogenannte "opt-out” Regelungen beim Impfen sind eine effektive Maßnahme (Chapman et al., 2010, 2016). Einige Menschen lassen sich nicht impfen, weil das Vereinbaren eines Termins mit Aufwand verbunden ist, "der Alltag dazwischen kommt" oder Impfen keine Priorität hat. Wenn der Termin jedoch gesetzt wird, dann ist das begründete Absagen eines solchen Termins ebenso mit Aufwand verbunden. Zudem kann das Setzen eines Termins durch andere eine Entlastung sein, wenn die Auseinandersetzung mit der Impfentscheidung als belastend empfunden wird. Gründe wie Vergessen oder Nachlässigkeit werden ausgeschlossen. Es wird zusätzlich die soziale Norm "Impfen ist der Standard" kommuniziert (Everett et al., 2015). Absagen sind bei opt-out Regelungen vermutlich vornehmlich noch von Personen zu erwarten, die eindeutig gegen das Impfen eingestellt sind (Yan \& Yates, 
2019) — dies sind laut Schätzungen der COSMO Studie bis zu 10\% der erwachsenen Bevölkerung zwischen 18 und 74 Jahren. Voraussetzungen für diese Intervention ist, dass bekannt ist, wer noch nicht über Impfschutz verfügt und diese Personen müssen erreichbar sein. Es muss auch damit gerechnet werden, dass Termine nicht abgesagt werden und einfach verfallen, was die Effizienz dieser Maßnahme unterminiert.

\section{Beratungspflicht: Wer sich nicht beraten lässt, erhält eine Strafe}

Eine verpflichtende Impf-Beratung von zertifizierten Impfberater:innen kann als Pflicht verhängt werden. Nach einer verpflichtenden Beratung ist die Entscheidung für oder gegen eine Impfung immer noch frei. Dies hat den Vorteil, dass die Entscheidung auf der Basis aktuellen wissenschaftlichen Wissens getroffen werden kann und jede:r sich einmal bewusst mit der Entscheidung auseinandergesetzt haben muss. Wer der Pflicht nicht nachkommt, hat mit Strafen zu rechnen. Eine Studie aus den USA (Omer et al., 2018), wo man durch einen Freistellungsantrag einer Impfung entgehen konnte, zeigt, dass eine Beratungspflicht bei Kinderimpfungen zu weniger Freistellungsanträgen und zu einer höheren Impfrate führte. Auch hat sich gezeigt, dass eine verpflichtende Beratung dazu beiträgt, dass sich keine ungeimpften Cluster bilden können. Die Beratungspflicht könnte auch mit der zuvor ausgeführten Terminpflicht kombiniert werden: um einen automatisch vergebenen Impftermin abzusagen, müssen die Personen vorher verpflichtend ein Beratungsgespräch wahrnehmen. Für die Beratungspflicht gilt ebenso die Voraussetzung, dass bekannt sein muss, wer noch nicht über Impfschutz verfügt und dass diese Personen erreichbar sein müssen. Ferner sollten Beratende zertifiziert werden, damit für impfkritische Ärzt:innen kein Geschäftsmodell mit dem Ausstellen von Beratungszertifikaten entsteht.

\section{Impfpflicht: Wer sich nicht impfen lässt, erhält eine Strafe}

Menschen werden mit einer Impfpflicht nicht zu einer Impfung gezwungen. Stattdessen könnten analog zur Masernimpfpflicht ungeimpfte Personen mit einer Geldstrafe und einem Berufsverbot belegt werden: sie dürften keiner beruflichen Tätigkeit mit körperlichem Kontakt mehr nachgehen. Verschiedene Begründungen für Ausnahmen könnten geltend gemacht werden. Die strengste Variante sind ausschließlich medizinische Gründe (wie bei der Masernimpfpflicht). In anderen Ländern werden auch religiöse oder weltanschauliche Gründe zugelassen. Je mehr Ausnahmen möglich sind, desto geringer ist auch die Effektivität der Impfpflicht (Omer et al., 2012). In manchen Ländern wie Australien wird der Bezug z.B. von Kindergeld an bestimmte Impfungen der Kinder geknüpft; Ausnahmen aus weltanschaulichen Gründen wurde von der Liste der möglichen Ausnahmen entfernt (Brewer et al., 2017). Regelungen, die ausschließlich medizinische Ausnahmen zulassen, sind zwar kurzfristig erfolgreich, mittelfristig steigt aber die Anzahl der beantragten Ausnahmen (Delamater, 2019). Nicht-medizinische Ausnahmen können die Akzeptanz der Maßnahme also evtl. erhöhen. Bei nicht-medizinischen Ausnahmen sollte es aber aufwändig sein, sie zu erhalten (z.B. durch einen Arztbesuch oder Besuch des Gesundheitsamtes, bei dem nach erfolgter Aufklärung unter Angabe von Gründen schriftlich erklärt oder beantragt werden muss, dass von einer Impfung Abstand genommen wird; ggf. muss die Ausnahme wiederholt beantragt werden, usw.; Omer et al., 2012). Für die Einführung einer allgemeinen Impfpflicht gegen COVID-19 ist es notwendig, dass alle Personen, für die die Impfpflicht gilt, ihren 
Impfstatus nachweisen (z.B. bei der Krankenkasse) und fehlende Impfnachweise durch eine Sanktion bestraft werden. Hierbei ist zu diskutieren, ab welchem Alter die Impfpflicht gelten soll (nur für Erwachsene oder auch für Kinder unter 18, für die ein Impfstoff verfügbar ist). Forschungsbefunde aus der Gesundheitspsychologie zeigen, dass Eltern einer eigenen Impfung eher zustimmen als der Impfung ihrer Kinder (Dore et al., 2014; Rees et al., 2021) so wäre anzunehmen, dass die Akzeptanz einer Impfpflicht für Erwachsene höher ist als eine, die auch Kinder einschließt.

\section{Wann sollte eine Impfpflicht erwogen werden?}

Arbeiten aus den USA (Mello et al., 2020) haben Kriterien aufgestellt, die vor der Einführung einer Impfpflicht gegen COVID-19 erfüllt sein sollten:

- COVID-19 ist nicht ausreichend eingedämmt.

- Die Impfung ist für diejenigen bereits empfohlen, für die die Pflicht erwogen wird.

- Es ist ausreichend Impfstoff vorhanden.

- Verfügbare wissenschaftliche Erkenntnisse zur Wirksamkeit und Sicherheit wurden transparent kommuniziert.

- Der Staat hat eine Infrastruktur geschaffen, die den Zugang zur Impfung ohne finanzielle oder logistische Hindernisse ermöglicht und die die Entschädigung von schwerwiegenden Nebenwirkungen und die Echtzeitüberwachung von Impfstoffnebenwirkungen ermöglicht.

- In einer zeitlich begrenzten Evaluierungsphase hat sich gezeigt, dass die Impfquote unter vulnerablen Gruppen bei einer freiwilligen Regelung zu gering ist, um eine epidemische Ausbreitung zu verhindern.

Bevor eine Impfpflicht eingeführt wird, sollten diese Kriterien geprüft und transparent kommuniziert werden. Ferner ist zu prüfen, ob die Erfüllung aller Kriterien auf alle Teile der Bevölkerung zutrifft oder nur für Teilpopulationen.

\section{Argumente für eine Impfpflicht}

Die mit einer Impfpflicht verbundene Einschränkung der individuellen Entscheidungsfreiheit scheint aus verschiedenen Gründen gerechtfertigt. Einige der hier dargestellten Vorteile können jedoch auch als Vorteile einer hohen Impfquote - nicht der Impfpflicht als solchesgewertet werden. Da diese Vorteile aber nur sehr unwahrscheinlich durch eine freiwillige Regelung zu erreichen sind, werden sie zu Vorteilen einer Impfpflicht.

\section{(1) Eine Impfpflicht ist sozial und stärkt das Gemeinwohl}

Wenn infolge einer Impfpflicht fast alle Menschen geimpft sind, dann hilft das auch denjenigen, die sich selbst nicht ausreichend schützen können. Das können Personen sein, die selbst nicht geimpft werden können, weil sie beispielsweise an einer Immunsuppression leiden. Aber auch Geimpfte können vom Schutz durch die Gemeinschaft profitieren. Die Wirkung der Impfung kann bei älteren Menschen eingeschränkt sein, selbst nach einer Boosterimpfung. Und Menschen mit schwerwiegenden Erkrankungen, etwa Krebspatient:innen, sind zwar meist gegen das Coronavirus geimpft, wegen ihrer Erkrankung 
und das Immunsystem belastender Therapien jedoch häufig anfälliger für eine Coronainfektion (Sorscher, 2021). Wenn durch die Impfpflicht Infektionsketten unterbrochen werden, entlastet das auch die Krankenhäuser. Von der Rückkehr zum Normalbetrieb profitieren insbesondere die Beschäftigten auf den Intensivstationen, die seit Beginn der Pandemie immer wieder an und über der Leistungsgrenze arbeiten. Gleichzeitig müssen bei Entspannung der Lage keine Behandlungen mehr verschoben werden, weil die notwendigen Ressourcen durch Coronapatient:innen gebunden sind oder für sie freigehalten werden (vgl. Krankenhausstatistik 2020). Das bedeutet einen deutlichen Zugewinn an Lebensqualität und auch Lebenszeit für diejenigen, die in den letzten eineinhalb Jahren auf Operationen und Therapien verzichten mussten. Außerdem entlastet dies auch psychisch-bei steigenden Fallzahlen, drohenden Einschränkungen und vermehrter Unsicherheit ist wieder ein Anstieg in der psychischen Belastung zu verzeichnen (COSMO \#56). Zusammengefasst kann eine Impfpflicht durch eine relevante Erhöhung der Impfquote die Gesundheit und Gesundheitsversorgung aller Menschen in Deutschland sichern und das Wohlbefinden in der Gesamtbevölkerung steigern.

\section{(2) Eine Impfpflicht kann effektiv sein}

Die Effektivität der Einführung einer Impfpflicht ist nicht eindeutig belegt. Wissenschaftliche Untersuchungen aus anderen Ländern weisen aber darauf hin, dass im Fall einer bestehenden Impfpflicht die Impfquoten bei Kindern und Beschäftigten im Gesundheitswesen höher sind als ohne eine Impfpflicht (Hull et al., 2019; Lee \& Robinson, 2016; Pitts et al., 2014). Beispielsweise zeigt eine Studie über 29 europäische Länder (Vaz et al., 2020), dass die Kinderimpfquoten für Masern und Keuchhusten in Ländern, in denen eine Impfpflicht für diese Krankheiten besteht, höher liegen als in Ländern, in denen diese Impfungen freiwillig sind. Im breiteren internationalen Vergleich gibt es allerdings sowohl Länder, die ohne Impfpflicht hohe Impfquoten haben und Länder, die trotz Impfpflicht niedrige Impfquoten haben (SABIN Institute, 2019). Die Maßnahmen, um die Verletzung der Impfpflicht zu sanktionieren, variieren hierbei international stark und reichen vom Ausschluss von Bildungsmaßnahmen bis hin zu Freiheitsentzug (Omer et al., 2019). Es gibt auch hierbei keinen Zusammenhang zwischen der Härte, mit der eine Impfpflicht durchgesetzt wird, und der Impfquote. Jedoch zeigt sich, dass die Gestaltung der Ausnahme-Regelungen einen Einfluss auf die Akzeptanz und Effektivität der Impfpflicht hat (Omer, 2012).

\section{(3) Eine Impfpflicht gegen COVID ist aktuell akzeptiert}

Die bisher genutzten Maßnahmen zur Steigerung der Impfquoten haben sich als unzureichend erwiesen. Werden die schnelle oder kontrollierte, langsame Durchseuchung als Alternativen abgelehnt, so bleibt damit nur noch die Einführung einer Impfpflicht, um eine hohe Immunisierung zu erreichen. Natürlich sollte diese von der Mehrheit der Bevölkerung unterstützt werden. Aktuelle Daten der COSMO-Studie und anderer Befragungen zeigen, dass die Unterstützung einer allgemeinen Impfpflicht in den vergangenen Monaten zugenommen hat und inzwischen von der Mehrheit der deutschen Bevölkerung befürwortet wird (COSMO \#56 und hier). Auch unter Ungeimpften ist jüngst ein minimaler Anstieg zu beobachten - in einem offenen Antwortformat wurde die Impfpflicht zudem von 5\% der Ungeimpften als Grund genannt, der sie zum Impfen bewegen würde (COSMO \#48-51). 
Sollte die Maßnahme eingeführt werden, so sollte sie allerdings nach einem gewissen Zeitraum evaluiert werden, da sich Einstellungen z.B. durch verändertes Infektionsgeschehen, auftretende Nebenwirkungen o.ä. ändern können (Meier et al., 2019).

\section{(4) Eine Impfpflicht kann sich positiv auf die Gesellschaft auswirken}

Mit einer Impfung schützen Geimpfte nicht nur sich selbst, sondern auch ihr Umfeld. Ein vollständiger COVID-19 Impfschutz reduziert die Übertragung auf andere um 63\% (de Gier et al., 2021). Damit wird Impfen zum sozialen Vertrag: ich schütze Dich, Du schützt mich. Wer diesen sozialen Vertrag eingeht, der erwartet auch von anderen, dass sie sich daran halten (Korn et al., 2020). Ein sozialer Vertrag bringt eine moralische Verpflichtung zur Impfung mit sich. Auch die Weltgesundheitsorganisation WHO spricht davon, dass Impfen ein "Recht und eine Verantwortung" ist (European Vaccine Action Plan; WHO, 2014). Über diese moralische Verpflichtung wurde bislang wenig öffentlich geredet. Vielmehr verbreiten sich Narrative über Entscheidungsfreiheit und das Recht, auch ungeimpft zu bleiben. Freiheit beinhaltet jedoch neben Rechten auch moralische Pflichten-zum Beispiel die moralische Pflicht, einen sozialen Vertrag einzuhalten und Rücksicht und solidarisches Handeln anderer positiv zu erwidern. Erfüllen viele Menschen den sozialen Vertrag nicht, führt dies zu sozialen Spannungen (Korn et al., 2020). Beispiele hierfür zeigen sich in Konflikten zwischen Geimpften und Ungeimpften; verschiedene Medien schreiben bereits von einer "gesellschaftlichen Spaltung" (z.B. hier, hier und hier). Eine Pflichtregelung kann in einer solchen Situation als fairer wahrgenommen werden als eine freiwillige Regelung, wie sich am Beispiel der Maskenpflicht zeigte (Betsch et al., 2020). In der zitierten Studie führte eine Pflichtregelung auch zu weniger Stigmatisierung. Dies ist in ähnlicher Weise auch bei einer Impfpflicht zu erwarten. Eine Pflicht kann in der aktuellen Situation, in der ungeimpfte Personen aus Infektionsschutzgründen in ihren Rechten sichtbar eingeschränkt werden und gesellschaftliche Kosten ansteigen, sozialen Konflikte die Grundlage entziehen und Polarisierung aufgrund von relevantem Gesundheitsverhalten verringern (Betsch et al., 2021).

\section{(5) Eine Impfpflicht kann Vertrauen stärken}

Häufig wird darauf verwiesen, dass die Einführung einer Impfpflicht dazu führen kann, dass Ungeimpfte ihr Vertrauen in den Rechtsstaat und die Regierung verlieren. Tatsächlich ist das Vertrauen von ungeimpften Personen in staatliche Organe bereits gering (COSMO \#56). Dass durch den Verzicht auf eine Impfpflicht in dieser Gruppe wieder Vertrauen aufgebaut werden kann, ist unwahrscheinlich. Vielmehr sollte alles daran gesetzt werden, das Vertrauen der großen Gruppe der Geimpften nicht zu verspielen. Es wird immer schwieriger, geimpften Personen zu erklären, warum sie die Konsequenzen aus der geringen Impfquote mittragen sollen. Wenn der Staat es nicht schafft, die Pandemie unter Kontrolle zu bringen, obwohl mit der Impfpflicht eine geeignetes Mittel zur Verfügung stünde, birgt dies viel größeres Potential für einen nachhaltigen Vertrauensverlust. Wenn sich mehr Menschen impfen lassen und in der Folge erkennen, dass die Impfung nicht mit den befürchteten Nebenwirkungen einhergeht, sondern vielmehr zu einem Rückgang der Infektions- und Sterbezahlen und einer Rücknahme von einschränkenden Maßnahmen wie etwa Kontakt- und Zutrittsbeschränkungen führt, könnte dies langfristig sogar das Vertrauen in Impfungen allgemein erhöhen. 


\section{Mögliche Nachteile einer Impfpflicht}

\section{(1) Eine Impfpflicht entbindet nicht von der Aufklärung}

Eine Impfpflicht wird voraussichtlich (zumindest kurzfristig) nicht dazu führen, dass sich die Einstellung bislang Ungeimpfter zur Impfung ändert, denn häufig vorhandene Sicherheitsbedenken und Ängste werden durch sie nicht adressiert. Aktive und niedrigschwellige Aufklärung zu Sicherheitsbedenken (und Korrektur hier oft vorhandener Falschinformationen) ist daher eine absolut unerlässliche Maßnahme, die die Einführung einer Impfpflicht begleiten muss.

\section{(2) Eine Impfpflicht kann psychologische Nebenwirkungen haben}

Die Ergebnisse psychologischer Experimente zeigen außerdem, dass Menschen, die der Impfung gegen COVID-19 kritisch gegenüberstehen und eine Impfpflicht ablehnen, sich über die Einführung einer Impfpflicht ärgern und in der Folge zum Beispiel andere freiwillige Impfungen, z.B. gegen Grippe, eher auslassen wollen (Sprengholz et al., 2021a, 2021b). Außerdem sinkt ihre Bereitschaft zur Einhaltung geltender Abstands- und Hygieneregeln, etwa zum Masketragen und Abstandhalten. Eine Impfpflicht könnte sich demnach auch negativ auf das Pandemiegeschehen auswirken, zumindest wenn andere Maßnahmen (z.B. Masketragen) weiter dringend wichtig sind und die Gruppe der ablehnenden Personen sehr groß ist. Andere denkbare Folgen wäre eine Verstärkung der nächsten Grippewelle. Als Gegenmaßnahme hat sich in Studien die Kommunikation des sozialen Nutzens als sinnvoll erwiesen: wer über den Schutz anderer aufgeklärt wurde, empfand weniger Ärger durch die Pflicht im Vergleich zu den Personen, die nicht aufgeklärt wurden (Sprengholz \& Betsch, 2020). Ebenso kann auch eine Aufklärung über den Nutzen für die Wirtschaftskraft diesen Effekt abmildern (Sprengholz et al., 2021b).

\section{(3) Eine zielgruppenspezifische Impfpflicht könnte die Gesamt-Impfquote negativ beeinflussen}

Eine zielgruppenspezifische Impfpflicht, die also die Impfung nur für einen Teil der Bevölkerung verpflichtend macht (z.B. Personen über 60 Jahre, die besonders vulnerabel sind), kann ebenfalls zu Nachteilen führen. Wir wissen aus der Forschung zu Impfempfehlungen, dass eine zielgruppenspezifische Empfehlung zwar die Impfbereitschaft in der betreffenden Bevölkerungsgruppe erhöht. Jedoch senkt sie gleichzeitig die Impfbereitschaft in Bevölkerungsgruppen, für die es keine Empfehlung gibt (Böhm et al., 2017). In der zitierten Studie war die Impfbereitschaft in den Zielgruppen ohne Impfempfehlung sogar geringer, als in der Gruppe ohne zielgruppenspezifische Empfehlung. Unter der Annahme, dass eine teilweise Impfpflicht eine Extremform der Impfempfehlung darstellt, könnte man vermuten, dass eine zielgruppenspezifische Impfpflicht nachteilig für die Gesamtimpfquote ist. Wäre die Bevölkerungsgruppe, für die die Pflicht gilt, klein, so könnte die Gesamtimpfquote am Ende geringer ausfallen als ohne zielgruppenspezifische Impfpflicht. 


\section{(4) Eine Impfpflicht könnte "impffaul” machen}

Eine Pflicht könnte auch ein Signal für zukünftige Impfentscheidungen setzen, zumal in Deutschland (bis auf die jüngste Verpflichtung zur Masernimpfung) alle anderen Impfungen freiwillig sind. Eine Pflicht für eine kleine Anzahl an Impfungen kann signalisieren: "wenn es keine Pflicht gibt, dann ist es nicht so wichtig”.

\section{(5) Eine Impfpflicht braucht begleitende Maßnahmen zur Stärkung der Akzeptanz}

Einschränkend ist zu sagen, dass die genannten Nachteile vor allem innerhalb experimenteller Forschung mit simulierten Impfentscheidungen oder hypothetischen Szenarien aufgetreten sind. Dennoch sollten sie als mögliche "psychologische Nebenwirkungen" ernstgenommen und antizipiert werden. Entsprechenden Maßnahmen zur Aufrechterhaltung von Vertrauen in das Gesundheitssystem und die politischen Entscheider:innen sollten ergriffen werden.

\section{Impfpflicht jetzt designen}

Die Einführung einer Impfpflicht ist eine politische Entscheidung-keine wissenschaftliche - und sollte das letzte Mittel sein, wenn nicht damit zu rechnen ist, dass Impfquoten mit anderen Maßnahmen gesteigert werden können. Klar ist, dass bei dem Design und der Implementierung einer Impfpflicht viele Aspekte zu bedenken sind (für welche Zielgruppen soll sie gelten, welche Ausnahmen soll es geben, wie soll sie überprüft werden, wie sollen Verstöße sanktioniert und eine Strafe umgesetzt werden, wie verbreitet man relevante Informationen zur Begründung der Maßnahme ihrer Umsetzung, und wie können mögliche negative psychologische Folgen abgefedert werden). In diesem Artikel werden zu einigen Fragen Antwortmöglichkeiten eröffnet, jedoch sind die Einsichten vieler anderer Disziplinen notwendig, um weitere Antworten in wissenschaftlichen Erkenntnissen zu finden. Dieser Prozess wird Zeit in Anspruch nehmen und sollte voller Sorgfalt von einem interdisziplinären Team verfolgt und die Ergebnisse transparent kommuniziert werden. Am Prozess sollten auch Sozial- und Verhaltenswissenschaftler:innen sowie Bürger:innen beteiligt sein.

Möglich wäre auch, dass die Ziel-Impfquoten des RKI als Entscheidungsmarke ausgegeben werden: Werden bis zu einem Stichtag die mit noch festzulegender Methode gemessenen Impfquoten nicht erreicht, gilt danach die Impfpflicht. Reichen andere Maßnahmen ausweitere Beispiele folgen im nächsten Absatz - tritt die Impfpflicht nicht in Kraft. Eine gut durchdachte und rechtssichere, umsetzbare Regelung in der Schublade liegen zu haben, erlaubt eine evidenzbasierte Anpassung und Veränderung der Regulierungen-bis hin zu einer möglichen Impfpflicht—entsprechend der pandemischen Lage.

\section{Was weiter getan werden könnte, um Aufklärung und die Impfquote zu erhöhen}

Unabhängig davon, ob eine allgemeine Impfpflicht beschlossen wird oder nicht, sollten weiter effektive Maßnahmen in der Kommunikation und Aufklärung umgesetzt werden. Der folgende Abschnitt gibt hierzu einige Beispiele.

Ansätze der Massenkommunikation (z.B. Informationen auf Webseiten) sollten durch Ansätze der interpersonellen Kommunikation ergänzt werden. Durch Massenkommunikation 
erreicht man eine breite Öffentlichkeit. Diese Reichweite geht jedoch auf Kosten der Wirksamkeit. Angebote direkter Gespräche durch vertrauenswürdige Meinungsführer:innen in den Gemeinden, auf öffentlichen Plätzen und vor der Haustür sind sinnvoll, um verunsicherte Menschen zu erreichen, ihre Fragen zu beantworten und über Impfungen aufzuklären. Dies ist vor allem sinnvoll für Gruppierungen, die vom RKI als seltener geimpft identifiziert wurden (z.B. Personen mit Migrationshintergrund, Menschen im Alter zwischen 30 und 39 Jahren, Arbeitssuchende bzw. Leute in Kurzarbeit, Personen in Wohnorten mit unter 20.000 Einwohnern; COVIMO). Diese Ansätze werden von Parteien im Wahlkampf genutzt und sollten dringend mit gleicher Intensität betrieben werden, wenn es um den Gesundheitsschutz der Bevölkerung in einer Pandemie geht (über erfolgreiche derartige Aktionen wird z.B. hier und hier berichtet).

In der Massenkommunikation sollten nicht nur Regierungskanäle und regierungsnahe Sprecher:innen eingesetzt werden. Empfehlungen, Informationen und Aufrufe zur Impfung müssen in allen Gesellschaftsschichten ankommen und viele Personen ohne Impfschutz haben wenig Vertrauen in die Regierung ( mit breiten Zielgruppen explizit für das Impfen positionieren, über Falschinformationen aufklären (gute Ressource dafür z.B. $\underline{\text { hier) }}$ und regelmäßig neue Empfehlungen vermitteln und erklären. Regelmäßige Formate (wie "Börse vor 8", Wetter) könnten hierfür Beispiele sein.

Journalist:innen und Medien sollten auch einen Fokus auf die Krankheit legen. Täglich sterben hunderte Menschen und Berichte über Schicksale von Hinterbliebenen und oder Ärzt:innen/Pflegepersonal sind immer noch rar. Der Fokus vieler Presseberichte liegt aber auf Querdenker:innen und Berichten über unerwünschte Nebeneffekte von Impfungen. Dabei gerät der Blick weg von der Krankheit und das dramatische Ausmaß der eigentlichen Katastrophe rückt in den Hintergrund (Maurer \& Gutenberg, 2021).

Community building funktioniert nicht nur über Slogans. Menschen, die aktiv helfen wollen, die Pandemie zu beenden, sollten in interpersonelle Kommunikationsprogramme eingebunden werden. Ebenfalls könnten wieder verstärkt Medizinstudierende rekrutiert werden, um über Impfen aufzuklären-z.B. in Schulen oder in Testzentren, die bei 3G Regelungen von Ungeimpften vermutlich auch häufiger genutzt werden. Ansätze "von der Bevölkerung für die Bevölkerung" sind keine Staatsansätze und können vom Vertrauensvorsprung in den Kommunikator profitieren. 


\section{Literatur}

Betsch, C., \& Böhm, R. (2016). Detrimental effects of introducing partial compulsory vaccination: experimental evidence. The European Journal of Public Health, 26(3), 378-381. https://doi.org/10.1093/eurpub/ckv154

Betsch, C., Korn, L., \& Böhm, R. (2021). Reply to Weisel: From polarization to vaccination and back. Proceedings of the National Academy of Sciences, 118(13).

https://doi.org/10.1073/pnas.2102717118

Betsch, C., Korn, L., Sprengholz, P., Felgendreff, L., Eitze, S., Schmid, P., \& Böhm, R. (2020). Social and behavioral consequences of mask policies during the COVID-19 pandemic. Proceedings of the National Academy of Sciences, 117(36), 21851-21853. https://doi.org/10.1073/pnas.2011674117

Böhm, R., Meier, N. W., Korn, L., \& Betsch, C. (2017). Behavioural consequences of vaccination recommendations: An experimental analysis. Health Economics, 26, 66-75. https://doi.org/10.1002/hec.3584

Brewer, N. T., Chapman, G. B., Rothman, A. J., Leask, J., \& Kempe, A. (2017). Increasing vaccination: putting psychological science into action. Psychological Science in the Public Interest, 18(3), 149-207. https://doi.org/10.1177/1529100618760521

Chapman, G. B., Li, M., Colby, H., \& Yoon, H. (2010). Opting in vs opting out of influenza vaccination. JAMA, 304(1), 43-44. https://doi.org/doi:10.1001/jama.2010.892

Chapman, G. B., Li, M., Leventhal, H., \& Leventhal, E. A. (2016). Default clinic appointments promote influenza vaccination uptake without a displacement effect. Behavioral Science \& Policy, 2(2), 40-50. https://behavioralpolicy.org/wpcontent/uploads/2017/06/chapman-web.pdf

de Gier, B., Andeweg, S., Backer, J. A., Hahné, S. J., van den Hof, S., de Melker, H. E., \& Knol, M. J. (2021). Vaccine effectiveness against SARS-CoV-2 transmission to household contacts during dominance of Delta variant (B. 1.617. 2), the Netherlands, August to September 2021. Eurosurveillance, 26(44), 2100977. https://doi.org/10.2807/15607917.ES.2021.26.44.2100977

Delamater, P. L., Pingali, S. C., Buttenheim, A. M., Salmon, D. A., Klein, N. P., \& Omer, S. B. (2019). Elimination of nonmedical immunization exemptions in California and schoolentry vaccine status. Pediatrics, 143(6), e20183301. https://doi.org/10.1542/peds.2018-3301

Dore, R. A., Stone, E. R., \& Buchanan, C. M. (2014). A social values analysis of parental decision making. The Journal of Psychology, 148(4), 477-504.

https://doi.org/10.1080/00223980.2013.808603 
Everett, J. A., Caviola, L., Kahane, G., Savulescu, J., \& Faber, N. S. (2015). Doing good by doing nothing? The role of social norms in explaining default effects in altruistic contexts. European Journal of Social Psychology, 45(2), 230-241. https://doi.org/10.1002/ejsp.2080

Hull, B., Hendry, A., Dey, A., Beard, F., Brotherton, J., \& McIntyre, P. (2019). Annual immunisation coverage report 2016. Communicable Diseases Intelligence, 43. https://doi.org/10.33321/cdi.2019.43.44

Korn, L., Böhm, R., Meier, N. W., \& Betsch, C. (2020). Vaccination as a social contract. Proceedings of the National Academy of Sciences, 117(26), 14890-14899. https://doi.org/10.1073/pnas.1919666117

Lee, C., \& Robinson, J. L. (2016). Systematic review of the effect of immunization mandates on uptake of routine childhood immunizations. Journal of Infection, 72(6), 659-666. https://doi.org/10.1016/j.jinf.2016.04.002

Maier, B., Burdinski, A., Klamser, P., Wiedermann, M., Brockmann, D., Jenny, M., Betsch, C. (in Vorbereitung). The pandemic is dominated by the unvaccinated population.

Maurer, M., \& Gutenberg, J. Eine empirische Studie zur Qualität der journalistischen Berichterstattung über die Corona-Pandemie. Rudolf Augstein Stiftung.

Meier, N. W., Böhm, R., Korn, L., \& Betsch, C. (2019). Individual preferences for voluntary vs. mandatory vaccination policies: An experimental analysis. European Journal of Public Health, 30, 50-55. doi.org/10.1093/eurpub/ckz181

Mello, M. M., Silverman, R. D., \& Omer, S. B. (2020). Ensuring uptake of vaccines against SARS-CoV-2. New England Journal of Medicine, 383(14), 1296-1299. https://doi.org/10.1056/NEJMp2020926

Omer, S. B., Allen, K., Chang, D. H., Guterman, L. B., Bednarczyk, R. A., Jordan, A., ... \& Salmon, D. A. (2018). Exemptions from mandatory immunization after legally mandated parental counseling. Pediatrics, 141(1), e20172364. https://doi.org/10.1542/peds.2017-2364

Omer, S. B., Betsch, C., \& Leask, J. (2019). Mandate vaccination with care. Nature, 571(7766), 469-472. https://doi.org/10.1038/d41586-019-02232-0

Omer, S. B., Richards, J. L., Ward, M. \& Bednarczyk, R. A. (2012). Vaccination policies and rates of exemption from immunization, 2005-2011. New England Journal of Medicine, 367, 1170-1171. https://doi.org/10.1056/NEJMc1209037 
Pitts, S. I., Maruthur, N. M., Millar, K. R., Perl, T. M., \& Segal, J. (2014). A systematic review of mandatory influenza vaccination in healthcare personnel. American Journal of Preventive Medicine, 47(3), 330-340. https://doi.org/10.1016/j.amepre.2014.05.035

Rees, F., Geiger, M., Lilleholt, L., Zettler, I., Betsch, C., Böhm, R., \& Wilhelm, O. (2021). Measuring parents' readiness to vaccinate themselves and their children against COVID-19. PsyArXiv, 21-11-06. https://doi.org/10.31234/osf.io/wrgce

SABIN Institute (2019). Legislative Approaches to Immunization Across the European Region. https://www.sabin.org/updates/resources/legislative-approaches-immunizationacross-european-region

Sorscher, S. (2021). Why it is our responsibility to speak out for a universal vaccination mandate. Cancer. https://doi.org/10.1002/cncr.33950

Sprengholz, P., \& Betsch, C. (2020). Herd immunity communication counters detrimental effects of selective vaccination mandates: Experimental evidence. EClinicalMedicine, 22, 100352. https://doi.org/10.1016/j.eclinm.2020.100352

Sprengholz, P., Betsch, C., \& Böhm, R. (2021a). Reactance revisited: Consequences of mandatory and scarce vaccination in the case of COVID-19. Applied Psychology: Health and Well-Being, 13(4), 986-995. https://doi.org/10.1111/aphw.12285

Sprengholz, P., Felgendreff, L., Böhm, R., \& Betsch, C. (2021b). Vaccination policy reactance: Predictors, consequences, and countermeasures. Journal of Health Psychology. https://doi.org/10.1177/13591053211044535

Vaz, O. M., Ellingson, M. K., Weiss, P., Jenness, S. M., Bardaj., A., Bednarczyk, R. A., \& Omer, S. B. (2020). Mandatory vaccination in Europe. Pediatrics, 145(2), e20190620. https://doi.org/10.1542/peds.2019-0620

Yan, H., \& Yates, J. F. (2019). Improving acceptability of nudges: Learning from attitudes towards opt-in and opt-out policies. Judgment and Decision Making, 14(1), 26-39. http://journal.sjdm.org/18/181018/jdm181018.pdf

Wichmann, O., Scholz, S., Waize, M., Schmid-Küpke, N., Hamouda, O., Wieler, L. H., \& Schaade, L. (2021). Welche Impfquote ist notwendig, um COVID-19 zu kontrollieren? Epidemiologisches Bulletin, 27, 3-13. https://doi.org/10.25646/8742

World Health Organization. (2014). European Vaccine Action Plan 2015-2020 (No. WHO/EURO: 2014-2227-41982-57703). https://apps.who.int/iris/handle/10665/340400

World Health Organization. (2017). Vaccination and trust: How concerns arise and the role of communication in mitigating crises. https://apps.who.int/iris/handle/10665/343299 